\title{
Unterrichtsklima, Partizipation und soziale Interaktion
}

\author{
Matthias v. Saldern
}

Schweer, M. (Hrsg.) Psychologie der Lehrer-Schüler-Interaktion. Opladen: Leske + Budrich, 2000. 159-176.

Ein wesentliches Merkmal der sozialen Interaktion zwischen Lehrkräften und Schülerinnen bzw. Schülern ist deren partizipative Charakter. Die Allgemeine Didaktik in ihrer Breite, einzelne didaktische Theorien im Besonderen sowie spezifische Unterrichtsmethoden thematisieren - wenn auch auf unterschiedlichen theoretischen und praktischen Niveau - die Rolle der Mitbestimmung im Unterricht. Dieses Merkmal scheint zusammenzuhängen mit dem sozialen Klima im Unterricht. Je nach Sichtweise ist Partizipation ein Merkmal des Unterrichtsklimas oder es steht außerhalb dieses Konstruktes und steht in Beziehung zur diesem. Im Folgenden wird erläutert, was Unterrichtsklima ist, welche Bedeutung die Partizipation im Unterricht spielt und wie beide Konzepte - auch über Erkenntnisse der Arbeits- und Organisationspsychologie - zusammengeführt werden können. Vor dem Hintergrund der empirischen Forschung über beide Konzepte wird deutlich, dass die Erfahrungen aus der Betriebs- und Organisationspsychologie für die Untersuchung der Lehrer-Schüler-Interaktion zumindest für den diskutierten Rahmen sinnvoll erscheint.

Dieser Bezug auf Erkenntnisse der Führungspsychologie des Betriebes hat keinen Selbstzweck. Er zeigt sehr deutlich auf, dass die Lehrer-Schüler-Interaktion und deren Erforschung theoretische und empirische Erkenntnisse aus Betrieb und Organisation mit einbeziehen muss. Dass der in der Schuldidaktik und Unterrichtsmethodologie Partizipation bereits mehrfach integriert ist, ist offenbar nicht zu den entsprechenden empirischen Untersuchungen geführt.

\section{Unterrichtsklima}

Unterrichtsklima ist eine spezifische Form des Sozialklimas. In der Literatur wird Sozialklima wie folgt beschrieben:

- Das Sozialklima beschreibt die Stimmung in einer Gruppe,

- es hat einen Bezugsrahmen (Schulklasse, Schule, Familie, Unterricht etc.),

- es hat relative Kontinuität über die Zeit (es ist nicht völlig stabil),

- es ist mehrdimensional (beschreibt also verschiedene Aspekte in der Gruppe gleichzeitig).

Unterrichtsklima ist bezogen auf eine bestimmte didaktische Situation, genannt Unterricht (und eben nicht nur bezogen auf Schulklasse oder gar Schule; zu anderen Deutungsmöglichkeiten des Begriffes siehe Eder, 1998, sie werden hier bewusst außer Acht gelassen.) Eine derartige Beschreibung ist allerdings rein formal. Es ist damit noch nicht gesagt, welche Inhalte damit verbunden sind.

Es gibt ein Fülle von Theorien zum Unterrichtsklima die hier nicht diskutiert werden sollen (siehe zfd. v. Saldern, 1987). Grundsätzlich arbeitet man in der Erforschung des Unterrichtsklimas mit mehreren theoretischen Annahmen, die als weitgehend bestätigt gelten können: 
1. Jeder Mensch hat ein Urteil über seine Umwelt, hier also: über seinen/ihren Unterricht.

2. Sozialklima basiert auf der Mehrheit der Mitglieder einer Gruppe. Kommen nahezu alle Mitglieder einer Gruppe zu einem ähnlich positiven Urteil, dann ist das Sozialklima auch positiv. Personen mit viel Macht (wie z.B. Lehrkraft) können das Sozialklima allerdings durch unbeliebte Entscheidungen oder unsachgemäßes Verhalten empfindlich stören.

3. Es handelt sich um überdauernde und typische Wahrnehmungen.

4. Es ist eine Aggregation über Zeit und verschiedene Situationen, erstellt aus Inferenzen gespeicherter situativer Einzeleindrücke.

5. Somit ist es so etwas wie die "Persönlichkeit" einer sozialen Situation. Der kollektive Charakter von Einzelwahrnehmungen wird über den Mittelwert erfasst. Es ist bis heute noch weitgehend ungeklärt, welchen Realitätsstatus der Mittelwert hat.

Um den Stellenwert der Klimaforschung heute einzuordnen, hilft ein Blick zurück: Die Entwicklung eines ehemals innovativen empirischen Forschungszweiges umfasst zu Beginn häufig die folgenden drei Phasen: (1) Entwurf einer theoretischen bzw. technologischen Programmatik; (2) Entwicklung geeigneter Erhebungsinstrumente; und (3) Nachweis der Bedeutsamkeit des Forschungsfeldes anhand der Analyse von relevanten

Außenzusammenhängen. Diese drei Arbeitsschritte kennzeichnen auch die Entwicklung der Klimaforschung seit Beginn der 50er Jahre: Im Vordergrund standen zunächst programmatische und rahmentheoretische Überlegungen sowie die Entwicklung von Messinstrumenten; anschließend wurde jeweils versucht, Zusammenhänge vor allem mit Schülerkriterien (Leistung, Persönlichkeit, Befinden) nachzuweisen. Allerdings liefen diese Schritte nur innerhalb einzelner Arbeitsgruppen tatsächlich zeitlich sequentiell: Seit der Jahrhundertmitte ist jedes größere Forschungsprogramm innerhalb der Sozialklimaforschung durch jeweilige Bemühungen in allen drei Bereichen gekennzeichnet.

\section{Partizipation}

Dem Begriff und Thema Partizipation kann man sich über drei Zugänge nähern: politisch, organisationspsychologisch und pädagogisch.

\section{a. Der politische Zugang}

Behrmann (1974, S. 307f) definiert Partizipation als Handeln, "mit dem jene, die formell nicht zu verbindlichen politischen Entscheidungen ermächtigt sind, versuchen, Entscheidungsprozesse zu beeinflussen." Die gängigen Kriterien für Partizipation wurden bereits von Habermas (1974, S. 138) wie folgt umschrieben: Aktivität, Informiertheit und Sachverständnis und affektive Bindung an das Geschehen.

Das National Council for the Social Studies hat schon 1962 als Untergruppierung des nordamerikanischen Lehrerverbandes Partizipation als Teilgebiet der Social Studies gefasst. Dazu gehören folgende Lernziele (Ackermann, 1974, S. 167f):

- Verschiedene Rollen innerhalb der Gruppe annehmen, die für den Fortschritt der Gruppe verantwortlich sind, wie z.B. die Rolle des ständigen störenden Fragers oder dessen, der die Diskussion zusammenfasst. 
- Parlamentarische Verfahrensweisen effektiv einsetzen.

- Differenzen innerhalb der Gruppe überwinden helfen.

- Kriterien und Verfahren zur Beurteilung des Gruppenprozesses vorschlagen und anwenden.

Partizipation im Klassensaal setzt voraus, dass die Kinder und Jugendlichen eine positive Beziehung zu diesem Konzept haben. In der Shell Jugendstudie 1997 wurde Antwort auf folgendes Statement erfragt: Mit dem Begriff 'Demokratie' verbinde ich eigentlich keine besondere Bedeutung. Durchschnittlich stimmten die Befragten weniger zu, wobei sich die Geschlechter nicht unterschieden. Je höher das Alter und je höher der Schulabschluss, desto geringer die Zustimmung. Dies ist an sich eine gute Ausgangslage, wenn man einmal von den Haupt- und Realschülern absieht, die zwischen "weniger Zustimmung" und "Zustimmung" liegen. Bei dem Statement Demokratie ist eine gute Staatsform, aber in Krisenzeiten ist sie nicht sehr effektiv liegen die Befragten aller Gruppen zwischen "weniger Zustimmung" und "Zustimmung". Diese skeptische Haltung findet sich bei dem Statement Demokratie ist das beste politische Modell, das es für einen Staat gibt nicht wieder: alle Befragten im Durchschnitt ebenfalls über alle Gruppen stimmen zu.

Trotz der manchmal skeptischen Haltung der Erwachsenen gegenüber den Jugendlichen scheinen diese der Demokratie im Makrosystem Gesellschaft näher zu stehen als oft angenommen. Dies kann man als günstige Voraussetzung werten, auch im Mikrosystem Schule partizipative Strukturen vorzufinden bzw. anzustreben.

b. der organisationspsychologische Zugang

Im Bereich der ABO-Psychologie wird Partizipation als besonders deutliches Merkmal der Ansätze der human-relations-Bewegung erkannt (Niederberger, 1991). Damit verbunden ist die Hinwendung zu mehr partizipativen und kooperativen Führungsstrukturen (v. Saldern, 1998). Der Begriff Partizipation wird dort durch Kooperation gefasst. Wunderer fasst die neun Merkmale kooperativer Führung (Wunderer, 1995, S. 1372) wie folgt zusammen:

- Ziel- und Leistungsorientierung,

- Funktionale Rollendifferenzierung und Sachautorität,

- Multilaterale Informations- und Kommunikationsbeziehungen,

- Gemeinsame Einflussausübung,

- Konfliktregelung durch Aushandeln und Verhandeln,

- Gruppenorientierung; partnerschaftliche Zusammenarbeit,

- Vertrauen als Grundlage der Zusammenarbeit,

- Organisations- und Personalentwicklung.

Die sich daraus ergebenen Dimensionen der Kooperativen Führung hat Wunderer wie folgt zusammengestellt (Die beiden folgenden Tabellen sind im Original belassen, da der Bezug zur Schule dem Leser unmittelbar deutlich werden wird.):

Tabelle 1: Dimensionen kooperativer Führung (Wunderer, 1995, S. 1372)

\begin{tabular}{|c|c|c|}
\hline $\begin{array}{c}\text { Zielorientierte soziale Einflussnahme zur } \\
\text { Erfüllung gemeinsamer Aufgaben }\end{array}$ & Ziel-Leistungs-Aspekt & \multirow{2}{*}{ Führung in Organisationen } \\
\cline { 1 - 2 } in/mit einer strukturierten Arbeitssituation & $\begin{array}{c}\text { Organisations-Aspekt } \\
\text { (Situationsgestaltung) }\end{array}$ & Qualität kooperativer Führung \\
\hline $\begin{array}{c}\text { unter wechselseitiger, tendenziell } \\
\text { symmetrischer Einflussausübung }\end{array}$ & $\begin{array}{c}\text { partizipativer Aspekt } \\
\text { (Machtgestaltung) }\end{array}$ \\
\hline
\end{tabular}


und konsensfähiger Gestaltung der Arbeitsund Sozialbeziehungen prosozialer Aspekt

(Beziehungsgestaltung)

Wunderer unterscheidet zusätzlich noch die partizipative und prosoziale Dimension, die sich wie folgt unterscheiden.

Tabelle 2: Dimensionen kooperativer Führung (Wunderer, 1995, S. 1378)

\begin{tabular}{|c|c|}
\hline \multicolumn{2}{|c|}{ Dimensionen Kooperativer Führung } \\
\hline $\begin{array}{c}\text { Partizipative Dimension } \\
\text { (interpositionale Machtgestaltung) } \\
\text { Teilhabe }\end{array}$ & $\begin{array}{c}\text { Prosoziale Dimension } \\
\text { (interpositionale Beziehungsgestaltung) } \\
\text { Teilnahme }\end{array}$ \\
\hline Informationsrechte/-pflichten & Kommunikation \\
Konsultationsrechte/-pflichten & Offenheit, Vertrauen \\
Begründungsrechte/-pflichten & Verständnis, Akzeptanz, Toleranz \\
Vorschlagsrecht & Zwischenmenschliche Orientierung \\
Mitentscheidung & Helfendes, solidarisches Verhalten \\
Kollegiale Entscheidung & Wechselseitigkeit \\
Vetorecht & Konstruktive Konfliktregelung \\
& Kompromiss- und Konsensfähigkeit \\
\hline
\end{tabular}

Der Definitionsansatz von Wunderer gilt derzeit als der elaborierteste. Dennoch stellt sich die Frage, wie Führung noch geschieht: Kooperative Führung muss - so die weitere Diskussion vielfach über Symbole erfolgen. Führung durch Symbole ist entpersonalisierte Führung. Sie ist in folgenden Situationen besonders wichtig (nach Weibler, 1995, S. 2022):

- Unsicherheit über das zu Erreichende,

- Zweifel bei der Bewertung von Zielen,

- niedrige Akzeptanz,

- Mitarbeiter inhaltlich von Vorgesetzten kaum noch zu steuern,

- fraglich, ob ethische Prinzipien das betriebliche Handeln legitimieren,

- Wunsch nach Veränderung der Identität der Organisation,

- Wunsch nach Änderung von Ansichten, Bedeutungen, Zielen,

- verminderter Kontakt zwischen Führer und Geführten,

- Wunsch nach Selbststeuerung des Geführten,

- Loyalität und Konsens wichtiger als Fachkenntnis,

- Ansprache an Kollektive, weniger an Einzelne.

Das Erstaunliche ist, dass derartige Führungsansätze im schulischen Bereich noch keinen Anklang gefunden haben. Zwar werden in der laufenden Diskussion zur Schulentwicklung andere Führungskonzepte diskutiert, sie erreichen aber in letzter Konsequenz weder kooperative noch die damit verbundene symbolische Führung. Dies, obwohl die genannte Beschreibung von Weibler ja durchaus auch in Schulen zu beobachten ist.

c. der pädagogische Zugang

Man wird die Frage stellen müssen, warum gerade Partizipation hier so besonders hervorgehoben wird, denn für die Erziehungswissenschaft ist Partizipation nur einer von vielen Werten. Neben den genannten theoretischen Konzeptionen stellt sich zudem natürlich die Frage, ob und inwieweit Partizipation in Unterrichtskonzeptionen Berücksichtigung findet. Ausgangspunkt der Analyse kann hier die Aufgabenstellung von Unterricht sein: Im Unterricht sollen Schüler lernen, Situationen durch Sinn zu bewältigen. Diese Aufgabe führte 
innerhalb der Didaktik zu einigen Kriterien eines guten Unterrichts, die man inzwischen durchaus als zeitlos ansehen kann:

- Situations-/Lebensbezogenheit

- Handlungsorientierung

- Wissenschaftsorientierung

- Prinzip des Exemplarischen

- Prinzip der Struktur

Gerade der erste Punkt (Situations-/Lebensbezogenheit) führt in unserer Gesellschaft zwangsläufig zur Berücksichtigung von Formen der Partizipation im Unterricht. Dies hat zum einen Gründe im politischen System (Mitbestimmung durch Wahl), aber auch durch rechtliche Rahmenbedingungen im Arbeitsalltag (Mitbestimmungsrechte). Aber auch die Wissenschaftsorientierung, die sich in der didaktischen Diskussion ursprünglich auf die Richtigkeit von Fachinhalten bezog, spielt im Falle der Partizipation eine verstärkte Rolle: Wenn man die Wissenschaftsorientierung von dem reinen Fachwissen erweitert auf Methoden- und Sozialkompetenzen, dann wird damit die didaktische Begründung für die Partizipation untermauert. Wenn man sich die Qualitätskriterien des guten offenen Unterrichts ansieht, dann gehören dazu: die Variabililität der gewählten Methoden, die Möglichkeit sich unkontrolliert zu bewegen und zu verhalten, der gegenseitige Respekt, die Moderation von Lernprozessen, die Sprachkultur und die Gestaltung der Umwelt.

Partizipation wurde auf der Ebene einzelner didaktischer Theorien neben anderen Ansätzen besonders eingebettet in Wolfgang Klafkis kritisch-konstruktiver Didaktik. Die Bezeichnung seiner Didaktik begründet der Autor durch das Ziel einer wachsenden Mitbestimmung und den engen Bezug zur Praxis. Klafki führt dazu noch den Emanzipationsbegriff der Frankfurter Schule mit ein: Emanzipation sei nur zu erreichen durch selbstbestimmtes exemplarisches Lernen. Und tatsächlich wird heute in dem typischen Unterrichtsdreieck (Lehrkraft, Schüler, Gegenstand) der schülerorientierte Unterricht wie in Abbildung $1 \mathrm{zu}$ sehen verortet.

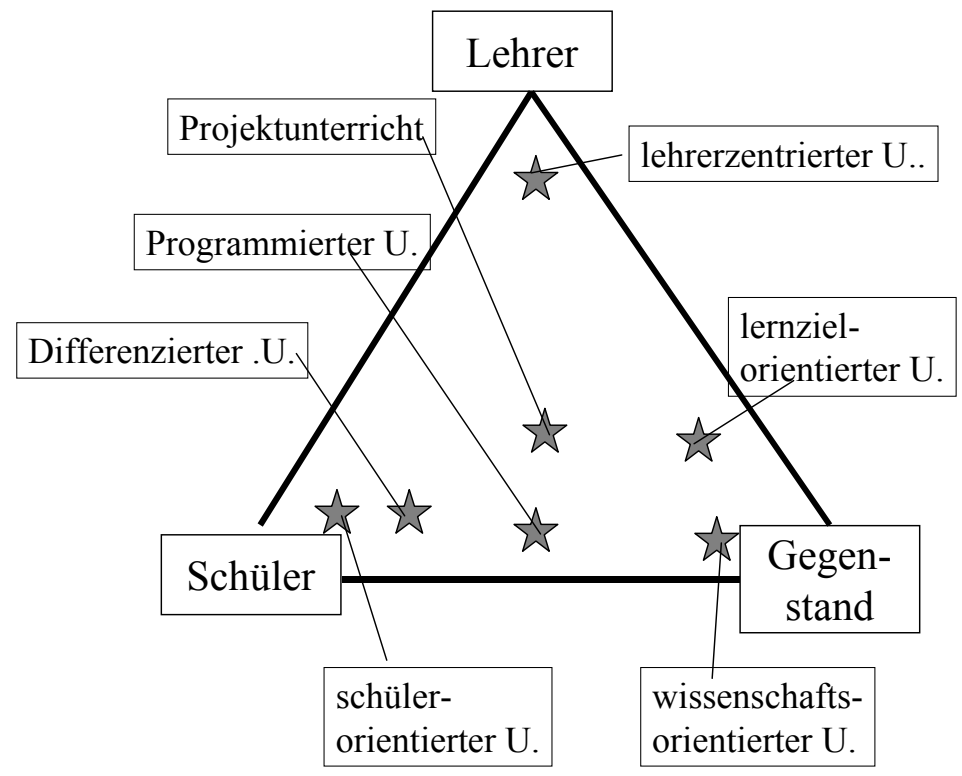

Abbildung 1: Unterrichtsformen im Dreieck Lehrkraft, Schüler und Gegenstand 
Der schülerrorientierte Unterricht geht letztlich auf das Ziel zurück, die Schülerinnen und Schüler zu demokratischen Verhaltensweisen zu erziehen. Die Rechtfertigung dazu liegt in den älteren Forschungen zum Erziehungsstil (zusammenfassend Brunner, 1998). Die Rolle der Partizipation in der Schulpädagogik wird heute allerdings unabhängig von allen theoretischen Konzeptionen in der Diskussion um den Offenen Unterricht in dem Mittelpunkt gestellt. Partizipation ist das Hauptanliegen dieser Unterrichtsform. Allerdings wird in der Diskussion die Frage deutlich, was Offenheit und Partizipation eigentlich bedeuten soll. Auch in Betrieben und Organisationen, die offene Führungsstile anstreben, wird ja keineswegs in allen Bereichen offen geführt. So stellt sich auch hier die Frage, was denn beim Offenen Unterricht eigentlich offen sein soll. Betrachtet man Unterricht ganz generell, dann ist Partizipation möglich bei Unterrichtsmethoden und -organisation, bei curricularen Fragen, bei dem Lehrer-Schüler-Verhältnis generell sowie bei der Gestaltung der Gesamtorganisation, in diesem Falle die Schule. Insofern ist Offenheit ein unscharfer Begriff, der einer Eingrenzung auf das bedarf, was offen sein. Dies gilt aber nun auch für die Partizipation: Worin und wobei sollen die Schülerinnen und Schüler mitbestimmen?

Es tun sich also eine Reihe von Fragen auf: Können die Schülerinnen und Schüler aufgrund ihrer Kenntnisse über Unterricht überhaupt in all diesen Dingen mitbestimmen? Ist der Mensch überhaupt in dem Maße an Mitbestimmung interessiert, wie hier unterstellt wird? Ist Partizipation in der Schule in ihrer ganzen Breite möglich und rechtlich zulässig?

Partizipation erzwingt neue Kooperationsformen (wie Offenen Unterricht) - zieht dies nicht eine verstärkte Bürokratisierung nach sich? Inwieweit wird das notwendige Eigeninteresse mit den Zielen der Organisation Schule in Einklang gebracht?

\section{Unterrichtsklima und Partizipation}

\subsection{Partizipation als Teil des Unterrichtsklimas}

Wie deutlich wurde, ist Partizipation neben den politischen und organisationspsychologischen Zugängen ein wesentlicher Bestandteil der pädagogischen Diskussion in Didaktik und Unterrichtsmethodologie. Man muss nun fragen, ob ein solches Kernelement von Unterricht Einzug in die Unterrichtsklimaforschung gefunden hat. Ein Weg zur Beantwortung dieser Frage ist die Analyse von den bisher konstruierten Fragebögen zum Unterrichtsklima.

Die US-amerikanischen Entwicklungen waren ursprünglich wegweisend auf dem Gebiet der Sozialklimaforschung gewesen. In den amerikanischen Verfahren wurde Partizipation, wenn auch nicht immer unter diesem Begriff, als wesentlicher Bestandteil des Unterrichtsklimas verstanden. Bereits im Learning Environment Questionnare (LEI; Anderson, 1973; Vorläufer: Classroom Climate Questionnaire; CCQ; Walberg, 1968) wurde eine Skala Democratic (Demokratische Entscheidungsfindung und Einflussstruktur) konstruiert. In der Classroom Environment Scale (CES; Moos \& Trickett, 1974) näherte man sich der Partizipation durch deren Gegenteil: die Skala Teacher Control soll das Ausmaß erfassen, in dem Schüleraktivitäten begrenzt werden. Aus der Kritik am CES und dem LEI entstand der Individualized Classroom Environment Questionnaire (ICEQ; Rentoul \& Fraser, 1979). Hier lautet die entsprechende Skala Independence (Möglichkeiten der Schüler, über ihr Verhalten selbst zu entscheiden). Bei der Analyse englischsprachiger Fragebögen muss man allerdings Vorsicht walten lassen, weil participation oft nur die Mitarbeit im Unterricht meint, und nicht die demokratische Beteiligung an alle Schul-. und Unterrichtsentscheidungen.

Derzeit allerdings scheint die amerikanische Forschung eher zu stagnieren, vorwiegend aus Gründen fehlender theoretischer Konzeptionen. Neuere Entwicklungen liegen dagegen für 
den deutschsprachigen Raum vor. Die Fragebogen, die im deutschsprachigen Raum entstanden sind, orientierten sich in der Anfangsphase an der US-amerikanischen Untersuchungen, wobei Partizipation nicht immer Berücksichtigung gefunden hat (z.B. beim Lernsituationstest von Kahl, Buchmann \& Witte, 1977). In den Konstanzer Schuluntersuchungen (Arbeitsgruppe um Fend) ergeben sich die folgenden Dimensionen: Inhaltsbereich (Selbständigkeitserwartungen, Leistungsdruck, Disziplindruck), Regulierungsbereich (Kontrollformen, Mitbestimmung), Beziehungsdimensionen (Engagement-Gleichgültigkeit, Vertrauen, Anonymität). Es ist also der Mitbestimmung ein klarer Platz zugewiesen. Im Fragebogen zum Unterrichtsklima (FUK; Dreesmann, 1980) heißt die entsprechende Skala Kooperation im Unterricht. Der Fragebogen ist nicht publiziert, aber es liegen einige Veröffentlichungen zu Untersuchungsergebnissen vor. Die Landauer Skalen zum Sozialklima (Lasso) sind als Testverfahren publiziert (v. Saldern \& Littig, 1986; Neuauflage 1996; Eder, 1998, dazu: "das elaborierteste Verfahren"). Bei diesem Instrument ist Partizipation erfasst mit der Skala Autoritärer Führungstil des Lehrers. In den Münchner Skalen zum Klassenklima (Pekrun, 1983) findet sich keine direkte Skala für Partizipation. Auf Itemebene entdeckt man den partizipativen Gedanken allerdings in den Skalen Unterstützung von Lehrerseite und Kooperation. Im Linzer Fragebogen zum Schul-und Klassenklima (Eder, 1990, 1998) ist es schließlich die Skala Mitsprache. Es wird also deutlich, dass Partizipation offenbar von den meisten Fragebogenkonstrukteuren berücksichtigt wurde.

\subsection{Befunde und Interpretationsprobleme}

Eine Übersicht über die Publikationen zum Unterrichtsklima kann hier nicht geleistet werden. Die folgenden durchaus nicht unabhängigen Bereiche spiegeln in etwa die Forschungsfragen wider:

- Einflüsse von Kultur, Epoche, Gesellschaft,

- Einflüsse von Schulsystem und Schulart,

- Einflüsse der Binnenorganisation von Schulen und Klassen (Klassenstufe, Schul- und Klassengröße, Schulfächer),

- Einflüsse der objektiven innerschulischen Umwelt (materielle Umwelt, Lehrermerkmale, Schülermerkmale, Interaktionsverhältnisse),

- Beziehungen zu anderen Umwelten von Schulmitgliedern (z.B. Familie),

- Schulklimawahrnehmungen: Prozesse, Inhalte, Strukturen,

- Einflüsse von Schulklima auf Schülerleistungen,

- Einflüsse von Schulklima auf Schülerpersönlichkeit und Befinden.

Die Befunde sind daher schwer zu integrieren. Dies liegt einmal an der Vielfalt der Dimensionierungen, was ja schon bei der Vorstellung der Fragebogen (s.o.) deutlich wurde. Den meisten Untersuchungen ist sicher der Themenkreis Leistung und Partizipation gemeinsam. Da aber die Unterrichtsmethoden und die damit einbezogenen Mitbestimmungsmöglichkeiten bei den meisten Untersuchungen fehlen, ist derzeit noch keine Aussage darüber möglich, inwieweit Partizipation z.B. Leistung beeinflusst. Hier sind die Erforschungen in Betrieben z.B. im Rahmen der Arbeitszufriedenheitsforschung weiter. Aber es gibt noch zwei weitere Gründe, warum die Ergebnisse zur Erforschung des Unterrichtsklimas schwer zu interpretieren sind. Zum einen sind die meist eingesetzten Fragebögen zwar ökonomisch und reliabel, sie bilden aber individuelle Kognitionen nicht ab. 
Zum anderen ist nicht deutlich, wie z.B. Zusammenhangmaße in der Unterrichtsklimaforschung zu interpretieren sind. Als Beispiel sei die Klimaskala Partizipation und die Zufriedenheit mit dem Unterricht herangezogen. Es sind bei Zusammenhangsstudien die Interpretation möglich, die in Abbildung 2 wiedergegeben sind.

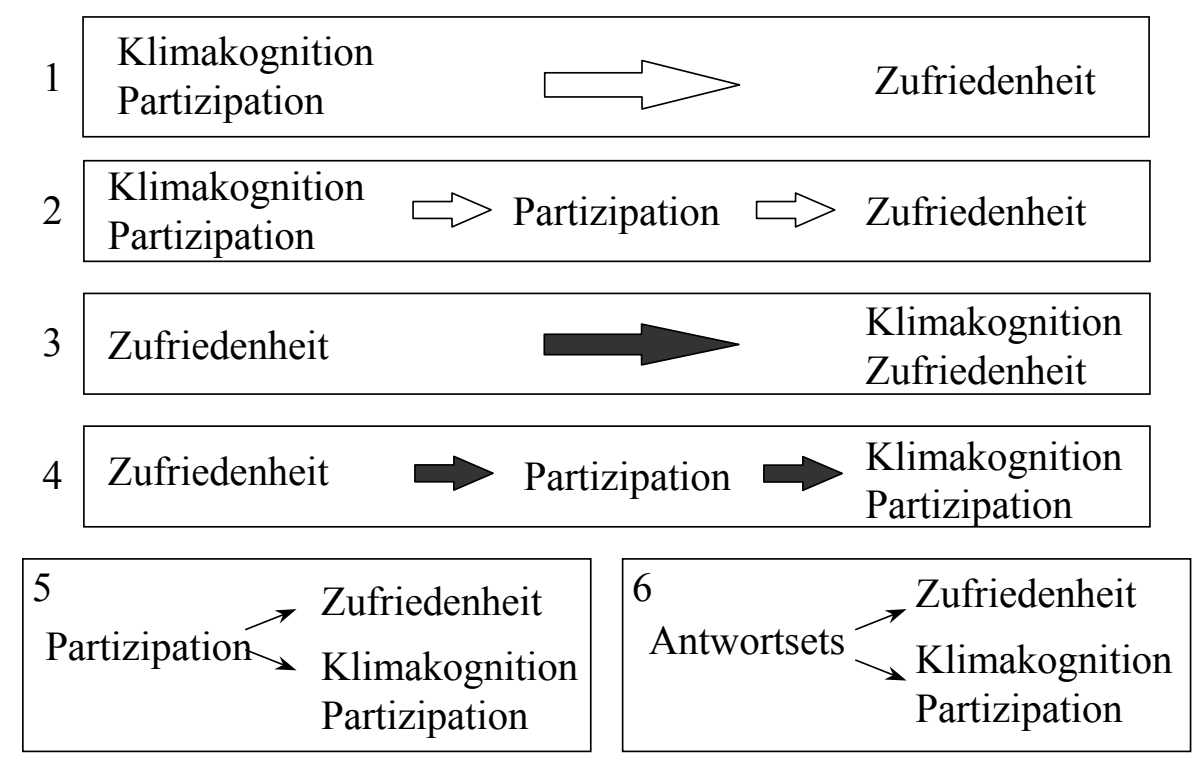

Abbildung 2: Mögliche Zusammenhänge

Zwar sind querschnittliche Zusammenhangsbefunde kausaler Interpretation gegenüber bekanntlich immer offen. Für zeitlich nicht gereihte Zusammenhänge von Klimakognitionen und anderen Größen aber stellt sich dieses Problem in schärferer Form als für andere pädagogisch relevante Zusammenhänge. Die folgenden Möglichkeiten der Interpretation eines Zusammenhangs zwischen einer Sozialklima- und einer Außenvariablen sind zu unterscheiden (vgl. auch Dreesmann et al., 1992, S. 671):

1. Die jeweilige Klimakognition wirkt direkt auf die jeweilige Bezugsvariable ein (Beispiel: Klimakognitionen von Partizipation bedingen Zufriedenheit).

2. Die Klimakognition wirkt indirekt auf die Bezugsvariable ein (Wahrnehmungen von Partizipation führen zur tatsächlichem Partizipation; diese erzeugt Zufriedenheit).

3. Die Bezugsvariable wirkt direkt auf die Klimakognition ein (Beispiel: zufriedene Schüler nehmen mehr Partizipation wahr).

4. Die Bezugsvariable wirkt indirekt auf die Klimakognition ein (Beispiel: Zufriedenheit führt zu Partizipation, diese wird wahrgenommen).

5. Die der Klimakognition zugrundeliegende Realität wirkt unabhängig voneinander sowohl auf die Klimakognition wie auf die Bezugsvariable ein (Beispiel: Partizipation führt zu kollektiven Partizipationswahrnehmungen und unabhängig davon zu individueller Zufriedenheit).

6. Die Erhebungen von Sozialklima und Bezugsvariable kovariieren aufgrund von Methodenvarianz (Beispiel: gemeinsame Antwortsets in Befragungen zu wahrgenommenem Partizipation und Zufriedenheit). 
Eine Schwäche der bisherigen Unterrichtsklimaforschung und damit ein Grund für manches Unbehagen liegt darin, zwischen solchen Interpretationsmöglichkeiten nicht systematisch differenziert zu haben. So ist es heute z.B. verfrüht, aus Korrelationen von Klimawerten und schulischen Leistungen (vgl. Haertel, Walberg \& Haertel, 1981) zu folgern, Sozialklima bedinge Leistung (und zwar selbst dann, wenn die jeweiligen pretest scores auspartialisiert wurden): Im Sinne der obigen Interpretation Nr. 5 nämlich muss sich dem außenstehenden Beobachter sofort die Annahme aufdrängen, dass faktische Unterrichtsprozesse der Leistung kausal zu Grunde liegen, woraus folgt, dass Korrelationen von Prozesswahrnehmungen und Leistung nur als Nebenprodukte solcher Effekte anzusehen wären. Unterrichtsklima käme in einem solchen Falle kein kausaler Stellenwert zu, sondern eher der Status eines Epiphänomens.

Da die pädagogische Psychologie auch an Schülereffekten interessiert sein muss, wurde dennoch in der Regel die Interpretation Nr. 1 (Sozialklimaeffekte auf den Schüler) bevorzugt (z.B. Helmke 1982; Pekrun, 1983). Seltener findet sich Interpretation Nr. 3

(Persönlichkeitseffekte auf Sozialklimawahrnehmungen; z.B. Littig \& v. Saldern, 1986). Die naheliegenden Interpretationsmöglichkeiten 4 und 5 (Sozialklima als Epiphänomen) hingegen wurden aus Gründen, die auf der Hand liegen, kaum beachtet. Eder (1990) könnte als Beispiel für Interpretation Nr. 4 genommen werden: Schüler/Lehrer mit bestimmten

Persönlichkeitsorientierungen 'erzeugen' Sozial- und Leistungsdruck.

Solange aber nicht (a) die betreffenden Daten zeitlich gereiht sind und (b) zur Ausschaltung der Interpretationen 2, 4 und 5 Daten zu den Bedingungen des Unterrichtsklimas nicht nur erfragt werden (also z.B. Beobachtungsdaten zu schulischen Interaktionen), handelt es sich hier bei Effektinterpretationen von Zusammenhangsdaten systematisch um Überinterpretationen. Daten zu Leistungs-, Persönlichkeits- und Befindenskorrelaten von Unterrichtsklima werten können damit vorläufig nicht als hinreichender Beleg für die Relevanz des Forschungsbereichs herhalten.

Der Stellenwert der Klimaforschung aber müsste sich daran zeigen, dass Klimakognitionen Wirkungen auf Leistung, Persönlichkeit und Befinden zeitigen, und zwar Wirkungen, die über direkte Effekte der faktisch vorhandenen Umweltverhältnisse hinausgehen. Zumindest müsste in diesem Sinne nachgewiesen werden, dass Klimakognitionen als Mediatoren von Wirkungen faktischer Umwelt anzusehen sind. Ein solcher, für die Relevanz dieses Forschungsfeldes existentieller Nachweis ist bisher nicht erbracht worden.

Wie stellt sich aber demgegenüber die Entwicklung der letzten 20 Jahre dar? Die Forschungen um das soziale Klima in Schulen, Klassen und im Unterricht verleiteten trotz der konzeptuellen Probleme fast zur Euphorie:

- Für die humanistisch gestimmten Psychologen erschloss sich mit dem Klimakonzept und den entsprechenden Instrumenten die Möglichkeit zu erfahren, was die Schüler über den Unterricht denken, um dann darauf eingehen zu können.

- Mit dem subjektiven Erleben als theoretische Basis schloss das Klimakonzept unmittelbar an die "kognitive Wende" in der Psychologie an, indem es die individuelle und kollektive Wahrnehmung und Verarbeitung von Umweltbedingungen in den Mittelpunkt rückte.

- Als erlebte Schulumwelt bot das Klimakonzept die Chance, den Trend zur Ökologie des Lernens theoretisch und praktisch umzusetzen und die schulischen Umfeldbedingungen in 
Modelle zur Erklärung schulischen Verhaltens, etwa der Schulleistung der Schulangst oder motivationaler Variablen, einbeziehen zu können.

\section{Die Erweiterung auf Kulturforschung}

Es darf allerdings bei allen Einschränkungen nicht übersehen werden, dass sich in den letzten Jahren sowohl bedeutsame theoretische Weiterentwicklungen als auch wichtige praktische Anwendungsversuche ergeben haben. Die Praxisrelevanz des Unterrichtsklmas ist ungebrochen: Die Fragebögen werden nach wie vor als Evaluationsinstrumente für pädagogische Interventionen benutzt. Die Ergebnisse bieten Hilfe bei schulischer Organisationsentwicklung und als Rückmeldeinstrumentarium für Lehrerkräfte. Dennoch scheint die theoretische Weiterentwicklung mehr Früchte zu tragen: Hier wurde der Begriff des Sozialklimas zu dem der Schulkultur weiterentwickelt (Hargreaves, 1995) und handlungstheoretisch interpretiert, was an der Definition von Schulkultur deutlich wird: Die Schulkultur ist die Gesamtheit der gewachsenen und durch die aktuelle Situation beeinflussten Wertvorstellungen, Verhaltensweisen und -routinen, Überzeugungen und Meinungen sowie Ordnungssysteme, Potentiale, Beziehungen und Gegebenheiten innerhalb einer Schule. Schulförmige Lebenswelt heute in ihrer schlechtesten Ausprägung kann wie folgt beschrieben werden: Standardisierung und Ökonomie des Raumes, Zeitökonomie (Tag, Woche, Jahr), Standardisierung der Lerninhalte, Kontrollcharakter, Praxisferne, ungewohnte Rituale, Mediatisierung und hierarchische Struktur

Die Diskussion um die Schulkultur trat die handlungsgeleitete und interpretative Bearbeitung und Verarbeitung der institutionell vorstrukturierten Aufgabe des 'Schulehaltens' auf Schulebene (jenseits von System- und Einzellehrer-Ebene) in den Vordergrund. Auf diese Weise konnte auch an die Tradition der Reformpädagogik wieder angeknüpft werden, in der Gestaltung des Gemeinschaftslebens, die Pflege des Schullebens und die Schaffung eines jugendspezifischen Lebensraumes schon einmal eine große Rolle gespielt haben, um der genannten schulförmigen Lebenswelt entgegenzutreten. Sie konnte aber durch neuere Entwicklungen auf dem Gebiet des kooperativen, partizipativen Führungsstils, der Personalvertretungsrechte, der Organisationskulturen in der Wirtschaft so weiterentwickelt werden, dass die Unterschiede zu unattraktiven Lernfabriken ohne Zielperspektiven mit ödem sozialen Leben, der ausschließlichen Konzentration auf korrekte Verwaltungsabläufe und minimalen Kommunikationsstrukturen deutlich in den Vordergrund traten und begrifflich fassbar wurden. Als zentrale Bedingungsgröße für eine gelungene Schulkultur, die vor allem von einem hohen Motivationsniveau charakterisiert ist, das eine proaktive und keine abwehrende Problembewältigungshaltung impliziert, hat sich dabei die Mentalität und pädagogische Grundhaltung der Lehrerschaft herauskristallisiert. In der praktischen Anwendung haben gerade in den letzten Jahren vielen gelungene Bemühungen gesehen, den schulischen Lebensraum zu gestalten, so dass sogar von einer gewissen schulpädagogischen Renaissance gesprochen werden kann. Dies ist aber auch unbedingt notwendig, wenn man die Verhaltensweisen von Schülerinnen und Schüler betrachtet, die deviantes Verhalten zeigen. Dazu gehört die destruktive sekundäre Anpassung (Zerstörung, Provokation, Schwänzen, Lernverweigerung, Flucht, Rückzug, usw.) ebenso wie die gemäßigte sekundäre Anpassung (Mogeln, Abschreiben, Schwindeln, geheucheltes Interesse, Streiche, Blödeleien, Nebenengagement, usw.). Ziel muss es also sein, partizipative Strukturen als Teil der Gruppenkultur zu implementieren. Eine Gruppenkultur ist ein Muster gemeinsamer Grundprämissen, das die Gruppe bei der Bewältigung ihrer Probleme externer Anpassung und interner Integration erlernt hat, das sich bewährt hat und somit als bindend gilt; und das daher 
an neue Mitglieder als rational und emotional korrekter Ansatz für den Umgang mit diesen Problemen weitergegeben wird.

Die Frage ist nun, wie man die Erforschung des Unterrichtsklimas unter besonderer Berücksichtigung partizipativer Strukturen durch eine Kulturforschung auffangen und weiterführen kann. Ausgangspunkt weiterer empirischer Untersuchungen können die Merkmale zur Beurteilung von Kulturen sein:

1. Prägnanz: Wie klar sind Orientierungsmuster und Werthaltungen? Starke

Organisationskulturen zeichnen sich durch klare Vorstellungen darüber aus, was

erwünscht ist und was nicht. Werte, Standards und Symbolsysteme sind relativ konsistent.

Weiter sind die kulturellen Orientierungsmuster relativ umfassend angelegt, um in vielen

Situationen den Maßstab setzen zu können. Der Kulturinhalt (anspruchsvoll, trivial, un-/ moralisch) selbst spielt für die Beurteilung der Stärke keine Rolle.

2. Verbreitungsgrad: Ausmaß, in dem die Beteiligten die Kultur teilen. Starke Organisationskulturen zeichnen sich durch ein hohes Maß an Homogenität aus.

3. Verankerungstiefe: Sie stellt darauf ab, inwieweit die kulturellen Muster internalisiert, also zum selbstverständlichen Bestandteil des täglichen Handelns geworden sind.

4. Persistenz: Sie ist als weiteres Merkmal eng mit der Verankerungstiefe verbunden.

Darunter versteht man die Stabilität der kulturellen Gestalt über längere Zeit hinweg.

Neben diesen Merkmalen einer (partizipativen) Kultur gibt es im Bereich der Lehrer-SchülerInteraktion ein bestimmtes Kommunikationsverhalten, dass typisch ist für den partizipativen Führungsstils der Lehrkraft: die Absprache.
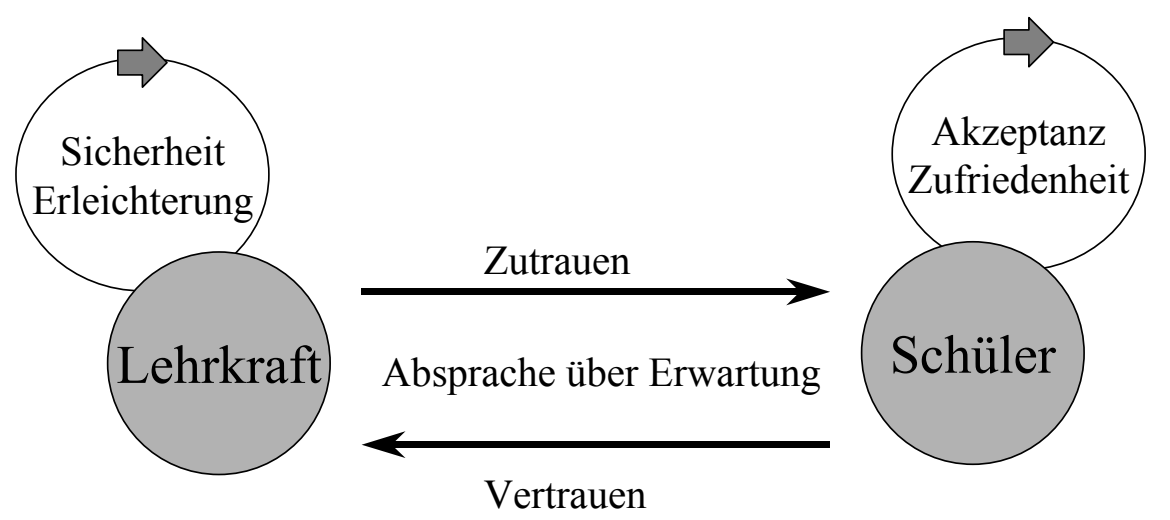

Abbildung 3: Absprachen zwischen Lehrkraft und Schüler

Wie in Abbildung 3 deutlich wird, sind Absprachen nicht nur zwingend notwendig im Sinn einer Regelfindung in der Schulklasse, sondern auch für die Gestaltung eines guten Unterrichtsklima. Absprachen sparen Nerven und ersetzen unausgesprochene Erwartungen. $\mathrm{Zu}$ den wesentlichen Merkmalen von Absprachen gehört, dass Zugeständnisse immer und leicht erfüllbar sein müssen und dass die Überprüfung gemeinsam stattfindet (zu Regeln der Führung durch Gespräche siehe v. Saldern, 1998a). Wenn man diesen Weg weiter denkt, dann kommt man zwangsläufig zu Studien, die in Betrieben und Organisationen durchgeführt wurden. 


\section{Literatur}

Ackermann, P. (Hrsg.) (1974): Politische Sozialisation. Opladen: Westdeutscher Verlag. Anderson, G.J. (1973): The assessments for learning environment: A manual for the Learning Environment Inventory and the My Class Inventory. Halifax/Canada.

Behrmann, G.C.( 1974): Bedingungen politischer Partizipation und die Grenzen politischer Bildung. In: Ackermann, P. (Hrsg.): Politische Sozialisation. Opladen: Westdeutscher Verlag, S. 304-323.

Brunner, E.J.( 1998): Lehrer-Schüler-Interaktion. In: Rost, D.H. (Hrsg.): Handwörterbuch Pädagogische Psychologie. Weinheim: Psychologie Verlags Union, S. 283-287.

Dreesmann, H. (1980): FUK - Fragebogen zum Unterrichtsklima. Landau. Unveröffentlichtes Manuskript.

Dreesmann, H.; Eder, F.; Fend, H.; Pekrun, R.; v. Saldern, M.; Wolf, B. (1992): Schulklima. In: Ingenkamp, K.; Jäger, R.S.; Petillon, H.; Wolf, B. (Hrsg.): Empirische Pädagogik 19701990 Weinheim: Deutscher Studien Verlag, S. 655-682.

Eder, F.(1990): Der Linzer Fragebogen zum Sozialklima (LFSK) Linz. Unveröffentlichtes Manuskript.

Eder, F.(1998): Schul- und Klassenklima. In: Rost, D.H. (Hrsg.): Handwörterbuch

Pädagogische Psychologie. Weinheim: Psychologie Verlags Union, S. 424-430.

Habermas, J.(1974): Politische Beteiligung, ein Wert "an sich". In: Ackermann, P. (Hrsg.):

Politische Sozialisation. Opladen: Westdeutscher Verlag, S. 138-142.

Haertel, G.D.; Walberg, H.J.; Haertel, E.H.(1979): Social - psychological environments and learning: A quantitative synthesis. San Francisco; AERA.

Hargreaves, D.H.(1995): School culture, school effectiveness and school improvement. In:

School Effectiveness and School Improvement, 6 (1), 23-46.

Helmke, A.(1982): Schulische Leistungsangst: Erscheinungsformen und

Entstehungsbedingungen. Konstanz.

Kahl, T.N. ; Buchmann, M. ; Witte, E.H.(1977): Ein Fragebogen zur Schülerwahrnehmung unterrichtlicher Lernsituationen. In: Zeitschrift für Entwicklungspsychologie und pädagogische Psychologie, 9, 277-285.

Littig, K.E. ; Saldern, M.v.(1986): Das Sozialklima in Schulklassen aus der Sicht von kooperativ bzw. kompetitiv orientierten Schülern In: Zeitschrift für Entwicklungspsychologie und pädagogische Psychologie, 18. 262-273.

Moos, R.H. ; Trickett, E.J.(1974): Classroom environment scale manual. Palo Alto:

Consulting Psychologists Press.

Niederberger, J.M.(1991): Organisationssoziologie und -psychologie von

Bildungsinstitutionen. In: Roth, L. (Hrsg.): Pädagogik. Handbuch für Studium und Praxis.

München: Ehrenwirth, S. 322-333.

Pekrun, R.(1983): Schulische Persönlichkeitsentwicklung. Frankfurt: Lang..

Rentoul, A.J. ; Fraser, B.J.(1979): Conceptualisation of enquiring-based or open classroom learning environment. In: Journal of curriculum studies, 1, 233-245.

Saldern, M.v.(1987): Sozialklima von Schulklassen. Frankfurt/M.: Lang.

Saldern, M.v.(1988): Grundlagen systemischer Organisationsentwicklung. Hohengehren:

Schneider.

Saldern, M.v.(1988a): Führen durch Gespräche. Hohengehren: Schneider.

Saldern, M.v. ; Littig, K.E.(1986): Lasso - Landauer Skalen zum Sozialklima. Weinheim:

Beltz.

Walberg, H.J.(1968): Structural and affective aspects of classroom climates In: Psychology in the Schools, 5, 247-253. 
Weibler, J.(1995): Symbolische Führung. In: Kieser, A.; Reber, G.; Wunderer, R. (Hrsg.): Handwörterbuch der Führung. Stuttgart: Schäffer-Poeschel, 2015-2026.

Wunderer, R.(1995): Kooperative Führung In: Kieser, A.; Reber, G.; Wunderer, R. (Hrsg.): Handwörterbuch der Führung. Stuttgart: Schäffer-Poeschel, 1369-1386. 